\title{
Inducing out-of-body experiences
}

\author{
Olaf Blanke and Gregor Thut
}

\section{Introduction}

In an out-of-body experience (OBE), people seem to be awake and feel that their 'self', or centre of experience, is located outside of the physical body. It is from an elevated extracorporal location that the subjects who undergo an OBE experience seeing their body and the world. The following example from Irwin $^{1}$ may illustrate what subjects experience during an $\mathrm{OBE}$ :

I was in bed and about to fall asleep when I had the distinct impression that 'I' was at the ceiling level looking down at my body in the bed. I was very startled and frightened; immediately [afterwards] I felt that, I was consciously back in the bed again.

An OBE is minimally defined by the presence of three phenomenological characteristics: disembodiment (location of the self outside one's body), the impression of seeing the world from an elevated visuospatial perspective (extracorporal, but egocentric visuospatial perspective) and the impression of seeing one's own body (autoscopy) from this elevated perspective. ${ }^{2-4}$ This is shown in Fig. 26.1.

Embodiment and egocentric perspective are processes mediating the self under normal conditions. Next to disembodiment, extracorporeal perspective and autoscopy, further characteristics of abnormal self processing during an OBE can be defined. Agency (or the feeling of being the agent of one's actions and thoughts) is localized at the position of the elevated, disembodied self. Moreover, the feeling of ownership (or of inhabiting one's body) is abnormal as the self that hovers over the autoscopic body is only rarely localized within a spatial entity or second body. ${ }^{1,3,5}$ Based on these characteristics, OBEs challenge the experienced spatial unity of self and body or the experience of a 'real me' that resides in one's body and is the subject of experience. ${ }^{6-9}$ This has also been suggested by psychologists ${ }^{1,3,10}$ and neurologists. ${ }^{4,11-13}$ These authors argued that OBEs are culturally invariant neuropsychological phenomena or deviant self models due to abnormal brain activation patterns whose scientific investigation might lead to a better understanding of the processes mediating the self under normal conditions. Understanding how the brain generates the abnormal self during OBEs is particularly interesting since OBEs are not only found in clinical populations, ${ }^{4,11-13}$ but also appear in approximately 10 per cent of the healthy population ${ }^{1,3}$ and have been described in the majority of the world's 


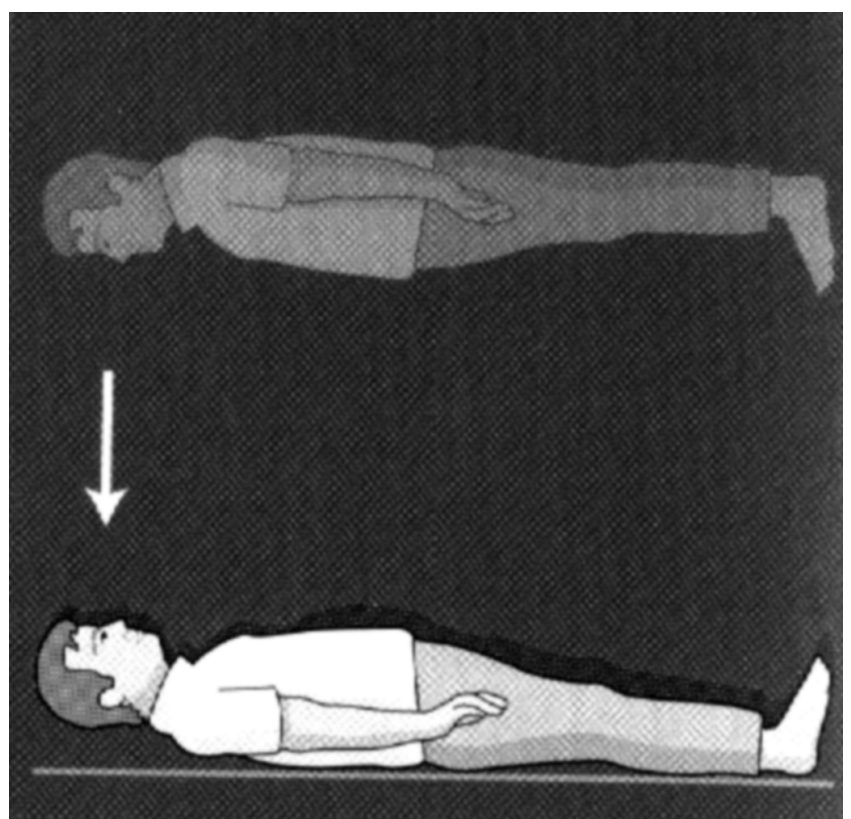

Fig. 26.1 Phenomenology of out-of-body experience (OBE). During an OBE, the subject appears to 'see' himself (bottom figure) and the world from a location above his physical body (extracorporeal location and visuospatial perspective; top figure). The self is localized outside one's physical body (disembodiment). The direction of the subject's visuospatial perspective during an OBE is indicated by an arrow (modified from Blanke $2004^{74}$ ).

cultures..$^{14}$ Moreover, several behavioural techniques have been described that are supposed to enhance the occurrence of OBEs. ${ }^{3}$ Although these techniques are quite diverse, they have some common characteristics and emphasize (1) a state of physical relaxation; (2) sensory deprivation; (3) mental imagery with respect to one's own body (bodyshape, size, weight or position); as well as (4) a state of elevated concentration and absorption as being important for the induction of an OBE. ${ }^{3}$ Yet, despite a large number of publications on OBEs, there are to date only few scientific investigations on the cognitive and neural basis of OBEs, probably because they generally occur spontaneously, are of short duration and happen only once or twice in a lifetime., ${ }^{2,3}$

The present review will focus on recent neurological and neuroimaging findings with respect to OBEs as well as on experimental techniques that have been used to induce OBEs artificially. This was done in order to investigate OBEs scientifically and to induce them experimentally in the research laboratory for the understanding of corporeal and self-awareness.

\section{OBEs induced by neurological disease}

The description of several neurological patients with OBEs due to brain damage has allowed a description to be made of the aetiology, associated phenomenology and 
anatomy of OBEs. ${ }^{4,11,13-16}$ OBEs have mainly been observed in patients with lesional and non-lesional epilepsy as well as migraine. Neurological authors have observed disturbed own-body processing in patients with OBEs. First the frequent association of vestibular sensations and OBE was reported, ${ }^{11}$ whereas others ${ }^{12}$ proposed that a paroxysmal vestibular dysfunction might be an important mechanism for the generation of OBEs. More recently, the importance of vestibular mechanisms in OBEs was underlined by their presence in all patients with $\mathrm{OBEs}^{4}$ and by the fact that vestibular sensations were evoked in one patient at the same site where higher currents induced an $\mathrm{OBE}^{17}$ (see below). In addition to vestibular disturbances, it has been reported that OBE patients may also experience paroxysmal visual body part illusions such as phantom limbs, supernumerary phantom limbs and illusory limb transformations either during the OBE or during other periods related to epilepsy or migraine.$^{4,11,17-19}$ Lesion analysis based on magnetic resonance imaging (MRI) showed involvement of the temporo-parietal junction in three OBE patients ${ }^{4}$ (Fig. 26.2; cases 1, 2a and 3). This was also found in another OBE patient ${ }^{20}$ (case 1). Moreover, OBEs can also be induced by electrical stimulation of the temporoparietal junction, pointing to the importance of this region in the generation of OBEs ${ }^{17}$ (see below). A recent review of all previously reported OBE cases of focal neurological origin (also including the earlier cases reported by Daly, ${ }^{21}$ Lunn $^{19}$ and Devinsky et al. ${ }^{11}$ ) found that OBEs were related in 75 per cent of cases to right hemispheric brain damage. ${ }^{16}$ Lesion overlap analysis of OBE patients with focal brain damage or focal electroencephalogram abnormalities is illustrated in Fig. 26.2.

Based on these neurological findings, a model of OBEs has recently been proposed ${ }^{4}$ suggesting that during an OBE the integration of proprioceptive, tactile and visual information of one's body fails due to discrepant central representations by the different sensory systems. This may lead to the experience of seeing one's body (autoscopy) in a position (i.e. on a bed) that does not coincide with the felt position of one's body (i.e. under the ceiling; see the above quoted example). As OBEs are also characterized by disembodiment and elevated visuospatial perspective, these authors speculated ${ }^{4}$ that an additional vestibular dysfunction is present in OBEs. They suggested that OBEs are related to an integration failure of proprioceptive, tactile and visual information with respect to one's own body (disintegration in personal space) and to a vestibular dysfunction leading to an additional disintegration between personal (vestibular) space and extrapersonal (visual) space. Both disintegrations were proposed to be necessary for the occurrence of an OBE. The neurological data also suggest that OBEs are due to a paroxysmal cerebral dysfunction of the temporo-parietal junction in a state of partially impaired consciousness or awareness due to epilepsy or migraine. ${ }^{4}$

Importantly, these clinical findings have allowed the complex phenomenon of the OBE to be linked with multisensory disintegration and deficient own-body processing at the temporo-parietal junction. This is not trivial, as these findings may help to demystify OBEs and facilitate the formulation of precise research hypotheses about the sensory, cognitive and neural mechanisms of OBEs. The neuroscientific investigation of OBEs may also turn out to be useful in defining the functions and brain structures mediating aspects 


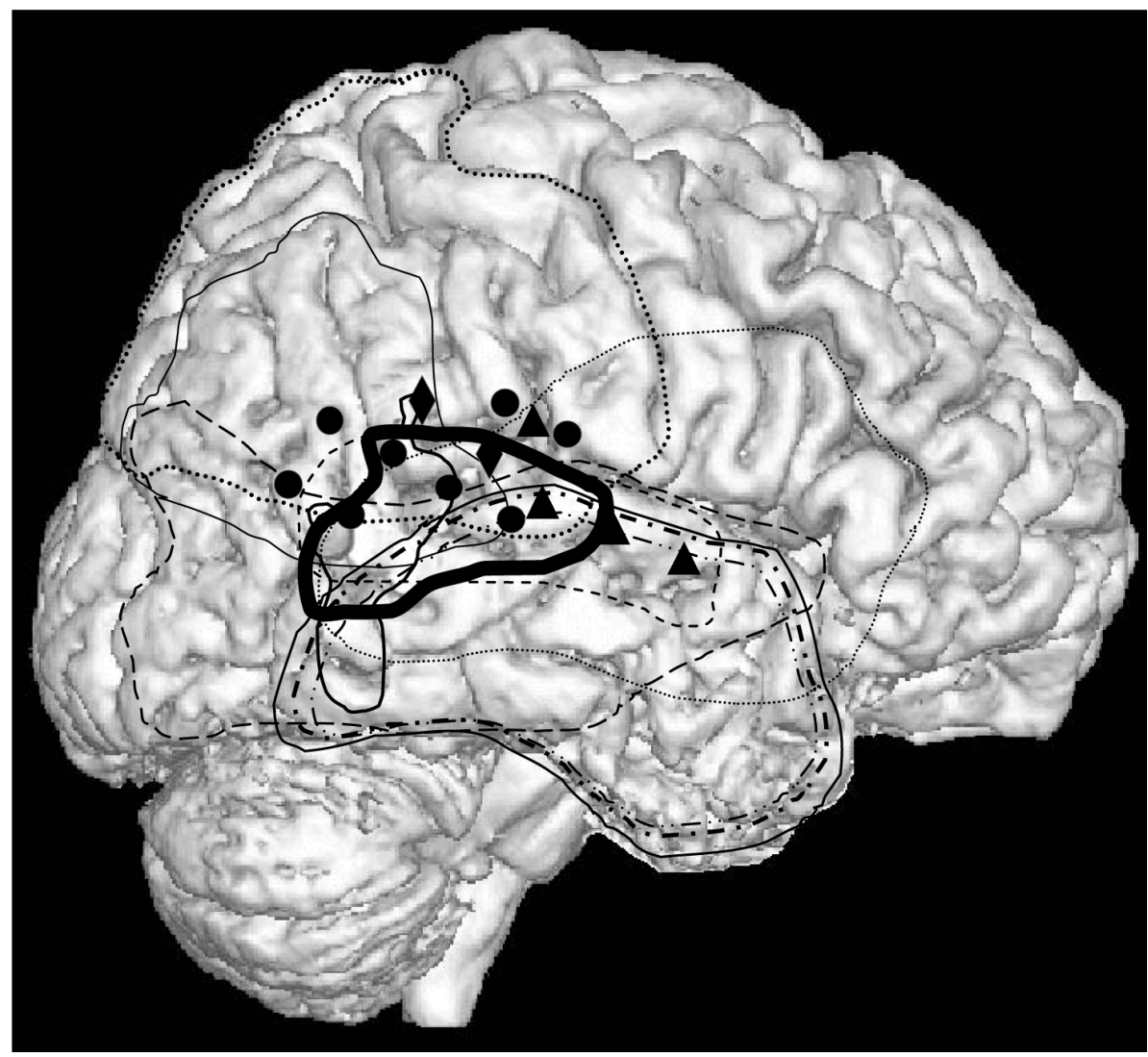

Fig. 26.2 Anatomy of out-of-body experience (OBE). MRI-based lesion overlap analysis of 13 previously reported OBE patients due to focal brain damage including patients from Penfield and Erickson ${ }^{22}(n=1)$, Penfield $^{23}(n=1)$, Daly $^{21}(n=1)$, Lunn $^{19}(n=1)$, Devinsky et al. ${ }^{11}(n=5)$, Blanke et al. ${ }^{4}(n=3)$ and Maillard et al. ${ }^{20}(n=1)$. The data for all patients are drawn on the MRI (right hemisphere) of one of the patients from the study of Blanke et al. ${ }^{4}$ (only two OBEs were due to left hemisphere interference). All lesions were estimated based on the anatomical results in the respective study (except the study by Blanke et al. where the lesions were transformed to Talairach space). Thus, when the lesion location (or the epileptic focus) was described as right temporal, we marked the whole temporal lobe for that patient; if the lesion was characterized as left fronto-temporal, parts of the fronto-temporal lobe were marked. The region of maximal overlap is indicated by the red area and was found on the right temporo-parietal junction.

of the normal self such as corporeal awareness, embodiment, egocentric visuospatial perspective and self-consciousness. ${ }^{9,16}$

\section{OBEs induced by electrical brain stimulation}

Before reviewing recent neuroimaging data about the temporo-parietal junction's implication in corporeal and self-awareness, we will first report what is known about the artificial induction of OBEs by focal electrical stimulation of the human brain. 
Recently, a patient has been reported ${ }^{17}$ in whom OBEs, vestibular sensations and visual body-part illusions were induced by direct electrical cortical stimulation of the right hemisphere. In this patient, who underwent intracranial pre-surgical epilepsy evaluation for intractable seizures, focal electrical stimulation at currents of $3.5 \mathrm{~mA}$ for $2 \mathrm{~s}$ induced an OBE that lasted for $2 \mathrm{~s}$ and was characterized by disembodiment, elevated visuospatial perspective and autoscopy. In this OBE, the patient experienced that her self was localized under the ceiling (almost touching it with 'her' back) and looking down on her body that was lying motionless on the bed. Repeated stimulations induced identical OBEs in the intrigued and surprised patient who had never experienced an OBE previously. Interestingly, smaller currents at this site $(2.0-2.5 \mathrm{~mA})$ led to vestibular sensations. ${ }^{17}$ The OBEs in this patient were induced whenever she looked straight ahead (without fixation of any specific object). If she fixated her outstretched arms or legs during electrical stimulation, she had the impression that the inspected body part was transformed, leading to the illusory, but very realistic, visual perception of limb shortening or illusory limb movement if the limbs were bent at the elbow or knee (currents of 4.0-4.5 mA). Finally, with closed eyes, the patient had neither an OBE nor a visual body-part illusion, but perceived her upper body as moving towards her legs. ${ }^{17}$ These data suggest that visual illusions of body parts and visual illusions of the entire body such as autoscopic phenomena might depend on similar functional and anatomical mechanisms, as argued by others. ${ }^{13,18}$

Wilder Penfield has described two further pre-surgical epilepsy patients (cases G.A. ${ }^{22}$ and V.F. ${ }^{23}$ ) who have reported experiences strongly resembling OBEs while undergoing electrical cortical stimulation. ${ }^{24}$ Below we discuss phenomenological, methodological, anatomical and epileptogenic differences and similarities between these patients and the above-described patient of Blanke et al. ${ }^{17}$ Regarding phenomenology, only little information has been provided by Penfield for patient V.F., ${ }^{23}$ who exclaimed 'Oh God! I am leaving my body'. It is thus not known what exactly this patient experienced or whether he was able to describe his experience more precisely (i.e. with respect to autoscopy, extracorporal visuospatial perspective or associated vestibular sensations). It was also not reported whether it was possible to reproduce the patient's experience. The sensation of 'leaving his body' was induced by electrical stimulation within the sylvian fissure at a depth of $2 \mathrm{~cm}$ on the surface of the superior temporal gyrus close to the insula. The region of stimulation was within the patient's epileptic focus where he had been operated on before. The stimulation that evoked the 'OBE' also elicited a concurrent seizure that was characterized initially by an unpleasant taste followed by swallowing movements, mental confusion and slow electroencephalogram waves of $4 \mathrm{~Hz}$. It was during this latter ictal period that the patient exclaimed that he was 'leaving his body'. Interestingly, stimulation at a site $1 \mathrm{~cm}$ posteriorly to the 'OBE'-inducing site elicited illusory whole0body movements described as if he would be standing up or spinning around (resembling the patient's habitual aura). The descriptions of patient G.A.'s experiences ${ }^{22}$ are even less similar to full-blown OBEs and were rather illusory whole-body and contralateral arm movements. Thus, G.A. exclaimed that 'I feel queer' and asked 'Am I here?' and 
described that she felt as if she was floating away and as if her left arm was moving. These experiences were reproducible, but induced at four different sites including the supramarginal gyrus and the superior temporal gyrus. G.A. suffered from focal epilepsy due to right hemispheric atrophy and local microgyria in the frontal, upper and lower postcentral areas associated with contralesional corporeal atrophy. It is important to note that there are not sufficient phenomenological details to classify these patients' experiences unambiguously as OBEs. In addition, both patients of Penfield might have had substantial cortical reorganization at the site whose stimulation resulted in an OBE since the epileptic focus was either very close to or directly at the stimulation site. This was not the case in the patient of Blanke et al. ${ }^{17}$ Despite these differences between the two patients of Wilder Penfield and the patient of Blanke et al., ${ }^{17}$ there are a number of striking phenomenological similarities including disembodiment, vestibular sensations and visual body part illusions. Furthermore, both previously reported cases of electrically induced 'OBEs' were observed after stimulation of the right hemisphere at the temporo-parietal junction, matching patient 3 of Blanke et al. ${ }^{17}$ Note that although the total number of stimulation-induced 'OBEs' is very low, it might be significant that OBEs have never been reported following electrical stimulation of the left hemisphere. Right hemisphere predominance is also suggested by neurological lesion data ${ }^{16}$ (see Fig. 26.2).

\section{Neurocognitive mechanisms of OBEs}

Neuroimaging studies support the role of the temporo-parietal junction in many of the functions that are relevant for or dysfunctional in OBEs. These are vestibular processing, multisensory integration as well as the perception of human bodies or body parts. The core region of the human vestibular cortex ${ }^{25,26}$ is situated at the temporo-parietal junction including the posterior insula, and brain damage in this area has been associated with vestibular sensations and dysfunctions. ${ }^{27,28}$ Several neuropsychological and neuroimaging studies suggest the implication of the temporo-parietal junction and cortical areas along the intraparietal sulcus in combining tactile, proprioceptive and visual information in a coordinated reference frame. ${ }^{29}$ Interestingly, the temporo-parietal junction codes multisensory conflict between visual and proprioceptive information about one's arm position as proposed in the above OBE model for the entire body. ${ }^{30}$ The temporo-parietal junction is also involved in many different aspects of processing with respect to the human body including the perception of body parts, ${ }^{31}$ the entire body ${ }^{32,33}$ as well as biological motion. ${ }^{34,35}$ Importantly, some of these 'visual body' areas are modulated not only by visually presented human bodies or body parts, but also by limb movements (without visual feedback), suggesting their role in multisensory own-body perception. ${ }^{33}$ The temporo-parietal junction has also been involved in cognitive functions that are closely linked to self processing and OBEs such as egocentric visuospatial perspective taking, ${ }^{36}$ agency (the feeling of being the agent of one's actions and thoughts) ${ }^{37-39}$ and self-other distinction (the capacity by which one distinguishes between oneself and other conspecifics). ${ }^{38-42}$ This is of relevance as during OBEs one's visuospatial perspective 
and one's sense of agency are localized at the position of the disembodied self that is hovering above the physical body. In other words, the self is experienced as looking at the (autoscopic) body from a third (or other) person's visuospatial perspective and position. Furthermore, the temporo-parietal junction is the classical lesion site in patients with visuospatial neglect, ${ }^{43}$ a clinical condition, which has been shown to disturb the patient's egocentric spatial relationship with extrapersonal space and visuospatial perspective taking. ${ }^{36}$ Neuroimaging studies in healthy observers have also revealed activation of the temporo-parietal junction during egocentric visuospatial perspective changes in healthy subjects. ${ }^{40,44}$ Moreover, it has been shown that mental activities such as agency and selfother distinction activate the temporo-parietal junction. ${ }^{45}$

The relationship of some of these aspects of the self, the three essential phenomenological characteristics of the OBE (disembodiment, visuospatial perspective and autoscopy) and own-body processing at the temporo-parietal junction has been investigated in a recent study by our team. ${ }^{46}$ We used a mental imagery task with respect to one's own body (modified from ${ }^{47,48}$ ). Schematic human figures in front or back view (see Fig. 26.3A) were presented to healthy subjects and to a patient with OBEs, who were asked to indicate whether the schematic figure's left or right hand was marked. The subject's were also instructed only to give their response after having imagined themselves in the depicted position and visual perspective of the figure.

In the healthy subjects, we observed a selective activation of the bilateral temporoparietal junction that was stronger and longer when subjects imagined the position and visual perspective that is generally reported by people experiencing spontaneous OBEs (front-facing figures; see Fig. 26.3B). Importantly, in the epileptic patient with OBEs originating from the temporo-parietal junction, the results revealed a functional activation of the seizure focus during mental transformations of her body and visual perspective mimicking her OBE percepts (see Fig. 26.3C) directly linking OBEs, mental own-body imagery and the temporo-parietal junction. Based on these results, we argued that the temporo-parietal junction might be a crucial structure for the conscious experience of the normal self mediating spatial unity of self and body and egocentric visuospatial perspective, and that impaired processing at the temporo-parietal junction may lead to the experience of abnormal selfs such as OBEs. ${ }^{46}$

In summary, although many other cortical areas such as prefrontal cortex, anterior cingulum, postcentral gyrus, precuneus, occipito-temporal junction, insula and superior parietal lobule $30,34,35,40,45,49$ have been shown to play a role in self processing, the reviewed neuroimaging data on body and self processing as well as our data on OBEs suggest that the temporo-parietal junction is a key neural locus for self processing that is involved in multisensory body-related information processing as well as in processing of phenomenological and cognitive aspects of the self. Interestingly, the above-mentioned studies show that techniques that have been used voluntarily to induce OBEs and the phenomenology of OBE may be combined with classical neuropsychological paradigms of mental imagery and neuroimaging methods to examine the neural correlates of OBEs and the self. Ideally, these techniques may also be combined to induce OBEs artificially 

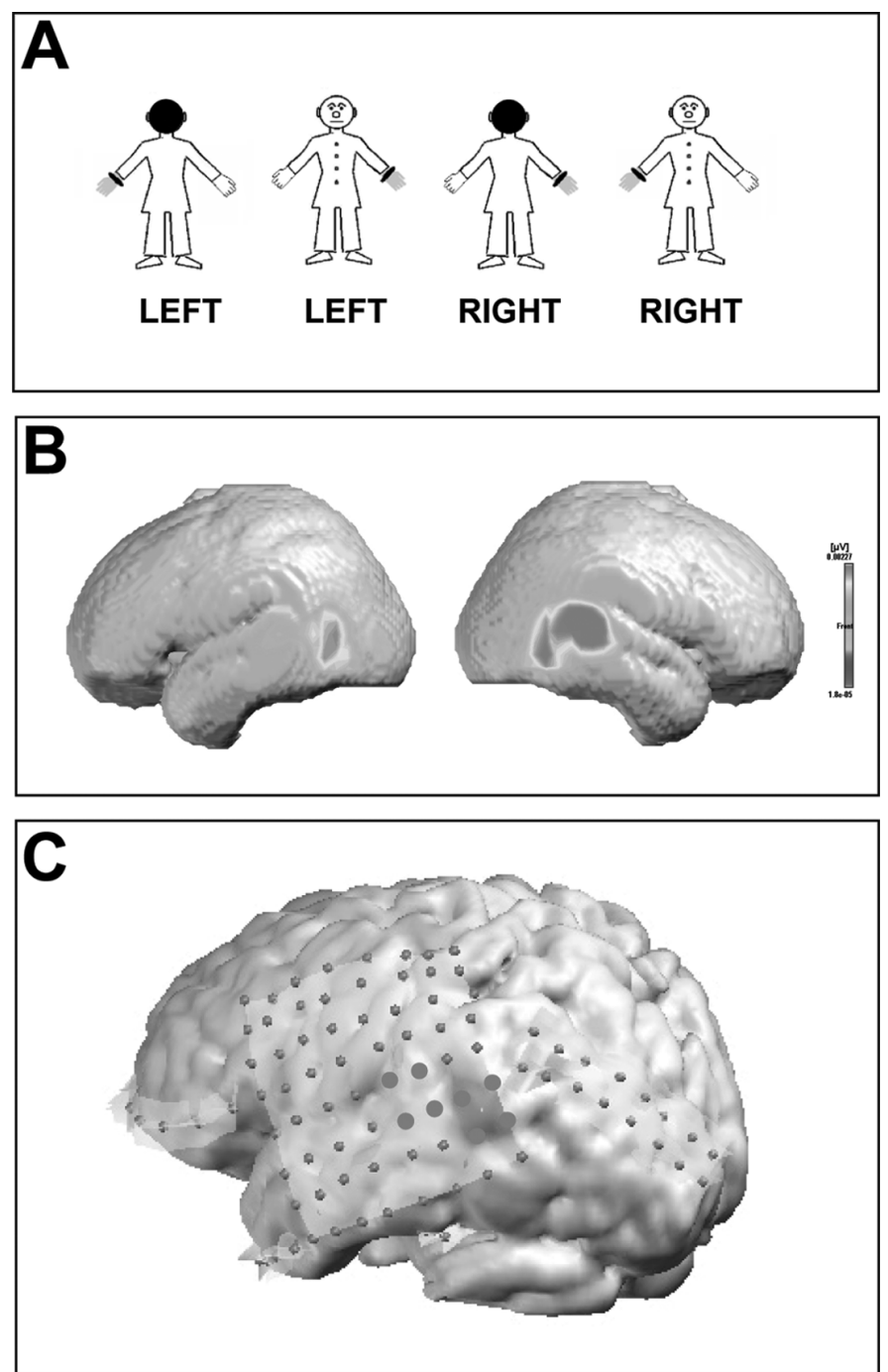

Fig. 26.3 Own-body mental imagery and electrical neuroimaging findings. (A) Four different stimuli as used in the own-body transformation task. Correct responses in the OBT task are indicated below each figure. Front-facing figures simulate the position and visuospatial perspective during spontaneous OBEs and led to longer reaction times in nornmal subjects (see Blanke et al. ${ }^{46}$ ). (B) A stable map topography was found from $>330-400 \mathrm{~ms}$ and only in the own-body transformation task (results not shown). This map's duration paralleled the behavioural reaction time differences in the experimental conditions in the own-body transformation task and led to an activation (as estimated by a linear inverse solution) of both temporo-parietal junctions with a right predominance (see Blanke et al. ${ }^{46}$ ). (C) MRI with the implanted electrodes overlying the lateral convexity of the left hemisphere. The epileptic focus, whose discharge induced an OBE, is indicated by eight red electrodes at the temporo-parietal junction. The figure also depicts the amplitude (in $\mu \mathrm{V}$ ) for all implanted electrodes during the own-body transformation task at $\sim 333 \mathrm{~ms}$ (blue depicts positive values and red negative values). The most prominent EPs at this latency were recorded over the temporo-parietal junction and partly overlapping with the epileptic focus. (See colour plate section) 
in healthy subjects as has previously been done for the induction of illusory phantom AU: Please limbs. ${ }^{50,51}$ Artificially induced OBEs (or illusory whole-body phantoms) ${ }^{52}$ would have the define EPs in great advantage of allowing the investigation of corporeal awareness and self processing Fig. 26.3 for the entire body. The possibility of scientifically inducing OBEs is discussed below with legend. respect to some behavioural conditions and transcranial magnetic stimulation (TMS).

\section{Combining TMS with cognitive neuroscience to induce OBEs}

TMS is a non-invasive technique to excite or inhibit transiently relatively circumscribed areas of the human brain. ${ }^{53}$ It is frequently used as a research tool in the study of human brain physiology and function. ${ }^{54,55}$ Originally conceived as an alternative method to the non-invasive, but painful technique of transcranial electrical stimulation, ${ }^{56}$ transcranial magnetic stimulation has also proven useful in cognitive neuroscience as an important extension of current neuroimaging procedures such as functional MRI (fMRI) or positron emission tomography (PET). ${ }^{55,57}$ During TMS, a strong, relatively focal and rapidly changing magnetic field is applied adjacent to the scalp, in order to stimulate electrically a given cortical area through electromagnetic induction. Because the magnetic field penetrates the cranium unimpededly, the stimulation is almost painless and normally well tolerated by the subjects. The induced current in the neural tissue underlying the magnetic stimulation coil transiently disrupts normal functioning of the stimulated area, which provides an important supplement to functional neuroimaging procedures such as fMRI or PET. Whereas functional neuroimaging reveals the areas that are active during a given task, disruption during TMS shows that a given area is not only active but also necessary for task performance. ${ }^{58}$

Using TMS in this disruptive mode, we have recently extended our finding of selective temporo-parietal junction activation when subjects mentally transform their body position into the position that is frequently reported by subjects with OBEs (front-facing figures; see above). We showed that interference with the temporo-parietal junction by TMS impaired this mental body transformation task in healthy subjects relative to magnetic stimulation of a control site ${ }^{46}$ (see Fig. 26.4A).

Furthermore, this functional interference by temporo-parietal junction stimulation was task specific, i.e. was not observed for mental transformations of the own-body position into back-facing figures (not matching the classically experienced 'OBE position', see Fig. 26.4B) and also not observed for a control task that implicated mental spatial transformations of external objects such as letters (adapted from classically used mental rotation paradigms; see Fig. 26.4C, D). Thus, interference by temporo-parietal junction stimulation with own-body transformations did not generalize to other, non-body spatial imagery tasks. It is worth mentioning that while TMS over the temporo-parietal junction selectively interfered with performance in the imagined body transformation task, TMS over the control site (consisting of the intraparietal sulcus region) impaired mental object-based transformations (see Fig. 26.4A versus C). This altogether was interpreted to support the notion that the temporo-parietal junction plays an essential role in the 

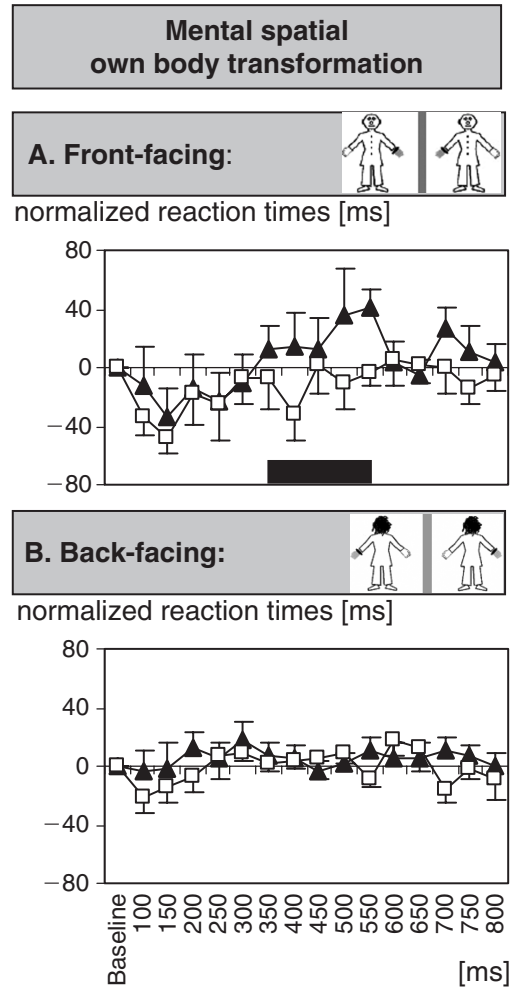
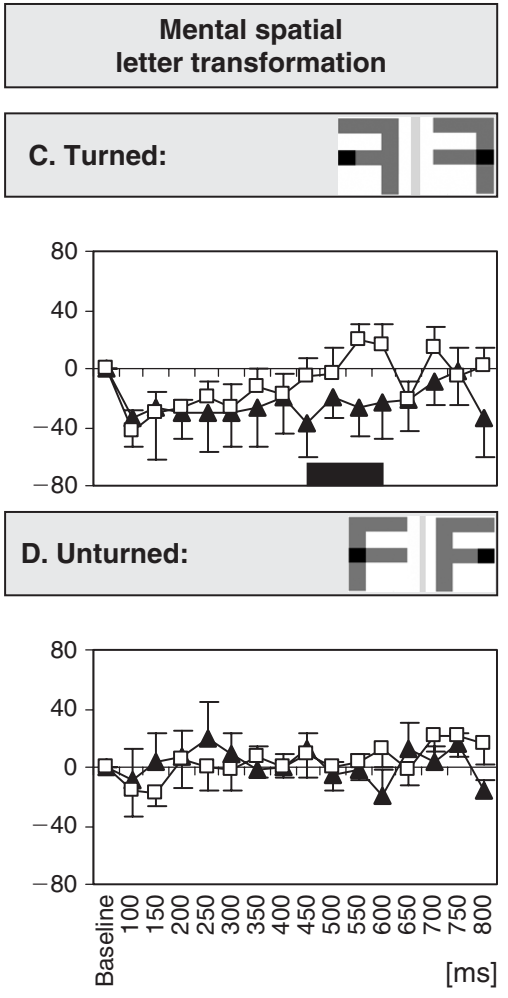

$\_$Temporo-parietal junction (TPJ)

$-\square-$ Control site = Intraparietal sulcus (IPS)

Fig. 26.4 Own-body mental imagery and TMS findings. TMS was used in its disruptive mode to probe the implication of the temporo-parietal junction in mental own-body transformations. A single TMS pulse was applied (1) over the temporo-parietal junction (black triangles) or (2) over a control site (intraparietal sulcus = white squares), while subjects were asked (i) mentally to transform their own body into a visually presented, schematic human figure (left panels) or (ii) to perform a control task consisting of mental spatial transformations of an external object, i.e. the letter ' $F$ ' (right panels). For further details, see text (and Blanke et al. ${ }^{46}$ ). Reaction times were recorded ( $y$-axis, reaction time normalized to baseline) as a function of 15 visual stimulus-TMS pulse onset asynchronies ( $x$-axis, 100-800 ms). (A) When subjects were mentally transforming their own body into front-facing figures, temporo-parietal junction stimulation at 350-550 ms interfered with task execution (prolonged reaction times) relative to TMS over the control site (intraparietal sulcus ). (C) When subjects were mentally transforming the letter ' $F$ ', an opposite effect was found. Intraparietal sulcus stimulation at 450-600 ms prolonged reaction times relative to TMS over the temporo-parietal junction. This indicates a double dissociation of mental ownbody and object-based transformations with respect to the site of TMS interference. (B and D) No effects were observed for mental spatial transformations into back-facing figures or of unturned letters. Data modified from Blanke et al. ${ }^{46}$ 
generation of OBEs as well as the mental imagery of one's own body position, i.e. in the updating of body position in space, egocentric perspective taking and corporeal awareness. ${ }^{46}$

The converging evidence that a temporo-parietal junction dysfunction might play a role in OBEs of neurological origin raises an interesting question. Is it possible to induce OBEs also in healthy volunteers through non-invasive TMS of the temporo-parietal junction? Of particular interest in this regard are situations in which TMS can be considered to operate in a productive rather than disruptive mode, i.e. when TMS is generating a discernible peripheral response or conscious percept. This is the case for TMS over the motor cortex, which induces a muscle twitch in the contralateral face, arm or foot depending on the site of TMS relative to somatotopic organization. ${ }^{59}$ Other productive effects of TMS include transient paraesthesia in the upper or lower extremities in response to stimulation of contralateral sensorimotor cortex ${ }^{59-61}$ as well as stationary or moving phosphenes in the peripheral or bilateral visual fields in response to stimulation of visual areas V1-V5, respectively. ${ }^{62-65}$ Finally, and most closely related to the present issue, TMS over motor cortex has also been reported to induce a 'sense of movement' when actual motor responses were ischaemically blocked. ${ }^{66,67}$ (Recently, the cortical origin of this kinaesthetic, body-part illusion has been questioned. ${ }^{68}$ ) To the best of our knowledge, TMS has never evoked productive effects when applied to higher order association cortex, such as the temporo-parietal junction, temporal cortex or the prefrontal cortex. However, given that direct electrical cortical stimulation of this area can elicit $\mathrm{OBEs}^{17}$ and given that OBEs can occur spontaneously in the healthy population, ${ }^{1,3}$ we argue that TMS over the temporo-parietal junction is also likely to evoke OBEs in healthy subjects when TMS is applied in specific experimental settings.

It has been suggested that OBEs result from a disturbance of on-line integration of various sensory inputs (proprioceptive, tactile, visual and vestibular) that normally provide a three-dimensional, dynamic representation of the body in space, combined with a prominent graviceptive vestibular dysfunction. ${ }^{4}$ We hypothesize that TMS could eventually interfere with such processes and hence lead to OBEs or related illusory sensations in conditions in which proprioceptive, tactile, visual and graviceptive vestibular inputs are weak or ambiguous. Interestingly, vestibulo-sensory illusions frequently occur during weightlessness on space missions and the low gravity phase of parabolic flights, and include the feeling of falling down, being upside-down, the sensation of self- and surround motion, or the sensation that fixed real visual targets move downwards. ${ }^{69-71}$ Also, temporary peripheral nerve block in neurologically healthy subjects can lead to a transiently impaired sense of ownership of the deafferented limb in spite of residual perception of position or posture or residual sensations of this limb..$^{72}$ Although weightlessness cannot be simulated in a laboratory, other techniques might be used to modulate vestibular and other sensory input and interfere with the subject's orientation regarding body position in space. For instance, mental relaxation techniques leading to a sense of lightness and floating might be used to modulate vestibular, proprioceptive and tactile processing (relaxation). A supine position on a surface that equally distributes 
gravity forces over the body might help to achieve this goal. For further disorientation in space, visual and auditory input should be prevented by blindfolding the subjects and using a soundproof room (sensory deprivation). Relaxation and sensory deprivation have previously been reported to facilitate the occurrence of OBEs, ${ }^{3}$ as introduced above. Moreover, additional weak vestibular stimulation (either physiological or galvanic) might be considered. Overall, this might facilitate the induction of discrepant central representations of the different sensory systems, suggested to be the neural basis of OBEs. Finally, there might be specific subpopulations of healthy subjects who are particularly prone to OBEs and hence its induction through TMS, given that some personality scores assessed through questionnaires on dissociative experience, hypnotic susceptibility and fantasy proneness have been found to relate to OBEs. ${ }^{5}$

\section{Conclusion}

In science, the most challenging phenomena are often the ones we take for granted in our everyday lives. An excellent example is the self and the experienced spatial unity between self and body that is challenged by OBEs. ${ }^{7,9}$ The evidence from neurological patients experiencing OBEs and thus the striking dissociation between self and body suggests that OBEs are culturally invariant phenomena, which can be investigated scientifically. The neuroscientific study of the self is in its infancy and there are currently no established models, very few data and often not even the vocabulary to describe neuroscientific notions of the self. ${ }^{73}$ The investigation of OBEs and related mechanisms at the temporoparietal junction might thus allow an improvement in our neuroscientific models of self and body. Although many other cortical areas are involved in self processing, recent neuroimaging studies suggest a key role for the temporo-parietal junction in OBEs and many aspects of body and self processing, such as the integration of multisensory bodily information, the visual perception of human bodies, biological motion perception, selfother distinction, agency and perspective taking. ${ }^{4,16,45}$ It is hoped that the experimental induction of OBEs and related experiences in healthy subjects will further our understanding of the central mechanisms of corporal and self-awareness much as previous research was successful with respect to the investigation of ownership of limbs by inducing an embodiment of artificial limbs in healthy subjects..$^{50,51}$

\section{References and notes}

1. Irwin HJ. Flight of mind: a psychological study of the out-of-body experience. Scarecrow Press, Metuchen, NJ. 1985.

2. Green CE. Out-of-body experiences. Hamish Hamilton, London. 1968.

3. Blackmore SJ. Beyond the body. An investigation of out-of-body experiences. Heinemann, London. 1982.

4. Blanke O, Landis T, Spinelli L, Seeck M. Out-of-body experience and autoscopy of neurological origin. Brain, 2004; 127:243-258.

AU: editors

names and year of publication?
5. Alvarado CS. Out-of-body experiences. In Varieties of anomalous experiences. American Psychological Association, Washington, DC. 183-218.

6. Shoemaker S. Self-reference and self-awareness. Journal of Philosophy, 1968; 65:555-567. 
7. Neisser U. The five kinds of self-knowledge. Philosophical Psychology, 1988; 1:35-59.

8. Gallagher S. Philosophical conceptions of the self: implications for cognitive science. Trends in Cognitive Sciences, 2000; 4:14-21.

9. Metzinger T. Being no one. MIT Press, Cambridge, MA. 2003

10. Palmer J. The out-of-body experience: a psychological theory. Parapsychology Review, 1978; 9:19-22.

11. Devinsky O, Feldmann E, Burrowes K, Bromfield E. Autoscopic phenomena with seizures. Archives of Neurology, 1989, 46:1080-1088.

12. Grüsser OJ, Landis T. The splitting of 'I' and 'me': heautoscopy and related phenomena. In Visual agnosias and other disturbances of visual perception and cognition. MacMillan, Amsterdam. 1991:297303.

AU: editors

names?

13. Brugger P, Regard M, Landis T. Illusory reduplication of one's own body: phenomenology and classification of autoscopic phenomena. Cognitive Neuropsychiatry, 1997; 2:19-38.

14. Sheils D. A cross-cultural study of beliefs in out-of-the-body experiences, waking and sleeping. Journal of the Society for Psychical Research, 1978; 49:697-741.

15. Brugger, P. Reflective mirrors: perspective taking in autoscopic phenomena. Cognitive Neuropsychiatry, 2000; 7:179-194.

16. Blanke O, Arzy S. (in press) The out-of body experience. Disturbed self processing at the temporoparietal junction. The Neuroscientist.

AU: in

17. Blanke O, Ortigue S, Landis T, Seeck M. Stimulating illusory own-body perceptions. Nature, 2002; 419:269-270.

18. Hécaen H, Ajuriaguerra J. L'Héautoscopie. In Méconnassiances et hallucinations corporelles. Masson, Paris. 1952:310-343.

19. Lunn V. Autoscopic phenomena. Acta Psychiatrica Scandinavica, 1972; 46:118-125.

20. Maillard L, Vignal JP, Anxionnat R, TaillandierVespignani L. Semiologic value of ictal autoscopy. Epilepsia, 2004; 45:391-394.

21. Daly DD. Ictal affect. American Journal of Psychiatry, 1958; 115:171-81.

22. Penfield W, Erickson TC. Epilepsy and cerebral localization. Charles C. Thomas, Springfield, IL. 1941:122-124; 261-265.

23. Penfield W. The twenty-ninth Maudsley lecture: the role of the temporal cortex in certain psychical phenomena. Journal of Mental Science, 1955; 101:451-465, 458-460.

24. Tong F. From Penfield to present. Trends in Cognitive Sciences, 2003; 7:104-106.

25. Lobel E, Kleine J, Leroy-Wilig A. Functional MRI of galvanic vestibular stimulation. Journal of Neurophysiology, 1999; 80:2699-2709.

26. Fasold O, von Bevern M, Kuhberg M, Ploner CJ, Vilringer A, Lempert T, Wenzel R. Human vestibular cortex as identified with caloric vestibular stimulation by functional magnetic resonance imaging. Neuroimage, 2002; 17:1384-1393.

27. Smith BH. Vestibular disturbances in epilepsy. Neurology, 1960; 10:465-9.

28. Brandt T. Central vestibular disorders. In Vertigo. Its multisensory syndromes. Springer, London. 2000:146-167.

press-please update.

AU: editors names?

29. Calvert GA, Campbell R, Brammer MJ. Evidence from functional magnetic resonance imaging of crossmodal binding in the human heteromodal cortex. Current Biology, 2000; 10:649657.

30. Leube DT, Knoblich G, Erb M, Grodd W, Bartels M, Kircher TT. The neural correlates of perceiving one's own movements. Neuroimage, 2003; 20:2084-2090.

31. Bonda E, Petrides M, Frey S, Evans A. Neural correlates of mental transformations of the body-inspace. Proceeding of the National Academy of Science, USA, 1995; 92:11180-11184.

32. Downing PE, Jiang Y, Shuman M, Kanwisher N. A cortical area selective for visual processing of the human body. Science, 2001; 293:2470-2473.

33. Astafiev SV, Stanley CM, Shulman GL, Corbetta M. Extrastriate body area in human occipital cortex responds to the performance of motor actions. Nature Neuroscience, 2004; 7:542-548. 
AU: in press-please update.

AU: in press-please update.
34. Grossman E, Donnelly M, Price R, Pickens D, Morgan V, Neighbor G, Blake R. Brain areas involved in perception of biological motion. Journal of Cognitive Neuroscience, 2000; 12:711-720.

35. Beauchamp MS, Lee KE, Haxby JV, Martin A. Parallel visual motion processing streams for manipulable objects and human movements. Neuron, 2002; 34:149-159.

36. Farrell MJ, Robertson IH. The automatic updating of egocentric spatial relationships and its impairment due to right posterior cortical lesions. Neuropsychologia, 2000; 38:585-595.

37. Farrer C, Frith CD. Experiencing oneself vs another person as being the cause of an action: the neural correlates of the experience of agency. Neuroimage, 2002; 15:596-603.

38. Farrer C, Franck N, Georgieff N, Frith CD, Decety J, Jeannerod M. Modulating the experience of agency: a positron emission tomography study. Neuroimage, 2003; 18:324-333.

39. Chaminade T, Decety J. Leader or follower? Involvement of the inferior parietal lobule in agency. Neuroreport, 2002; 13:1975-1978.

40. Ruby P, Decety J. Effect of subjective perspective taking during simulation of action: a PET investigation of agency. Nature Neuroscience, 2001; 4:546-50

41. Ruby P. Decety J. What you believe versus what you think they believe: a neuroimaging study of conceptual perspective-taking. European Journal of Neuroscience, 2003; 17:2475-2480.

42. Ruby P, Decety J. How do you feel versus how do you think she would feel? A neuroimaging study of perspective taking with social emotions. Journal of Cognitive Neuroscience, 2004; 16:988999.

43. Halligan PW, Fink GR, Marshal JC, Vallar G. Spatial cognition: evidence from visual neglect. Trends in Cognitive Science, 2003; 7:125-33.

44. Maguire EA, Burgess N, Donnett JG, Frackowiak RS, Frith CD, O'Keefe J. Knowing where and getting there: a human navigation network. Science, 1998; 280:921-924.

45. Decety J, Sommerville JA. Shared representations between self and other: a social cognitive neuroscience view. Trends in Cognitive Science, 2003; 7:527-533.

46. Blanke O, Mohr C, Michel CM, Pascual-Leone A, Brugger P, Seeck M, Landis T, Thut G. Linking outof-body experience and self processing to mental own body imagery at the temporo-parietal junction. Journal of Neuroscience (in press).

47. Ratcliff, G. Spatial thought, mental rotation and the right cerebral hemisphere. Neuropsychologia, 1979; 17:49-54.

48. Zacks J, Rypma B, Gabrieli JD, Tversky B, Glover GH. Imagined transformations of bodies: an fMRI investigation. Neuropsychologia, 1999; 37:1029-1040.

49. Zacks JM, Ollinger JM, Sheridan MA, Tversky B. A parametric study of mental spatial transformations of bodies. Neuroimage, 2002; 16:857-872.

50. Botvinick M, Cohen J. Rubber hands 'feel' touch that eyes see. Nature, 1998; 391:756.

51. Ehrsson HH, Spence C, Passingham RE. That's my hand! Activity in premotor cortex reflects feeling of ownership of a limb. Science, 2004; 305:875-877.

52. Brugger P. (in press) from phantom limb to phantom body. In G Knoblich, I Thornton, M Grosjean, M Shiffrar, ed. Perception of the human body inside out. Oxford University Press, Oxford.

53. Hallett M. Transcranial magnetic stimulation and the human brain. Nature, 2000; 406:147-50.

54. Chen R. Studies of human motor physiology with transcranial magnetic stimulation. Muscle Nerve, 2000; 9: S26-32.

55. Walsh V, Cowey A. Transcranial magnetic stimulation and cognitive neuroscience. National Neuroscience Reviews, 2000; 1:73-79.

56. Barker AT, Jalinous R, Freeston IL. Non-invasive magnetic stimulation of human motor cortex. Lancet, 1985; 1:1106-1107.

57. Pascual-Leone A, Walsh V, Rothwell J. Transcranial magnetic stimulation in cognitive neurosciencevirtual lesion, chronometry, and functional connectivity. Current Opinion in Neurobiology, 2001; 10:232-237. 
58. Theoret H, Pascual-Leone A. Transcranial magnetic stimulation and the study of cognition. In K Hugdahl, ed. Experimental methods in neuropsychology, Kluwer Academic Publishers, Boston. 2003:173-195.

59. Amassian VE, Cracco RQ, Maccabee PJ, Cracco JB, Henry K. Some positive effects of transcranial magnetic stimulation. Advanced Neurology, 1995; 67:79-106.

60. Amassian VE, Somasundaram M, Rothwell JC, Britton T, Cracco JB, Cracco RQ, Maccabee PJ, Day BL. Paraesthesias are elicited by single pulse, magnetic coil stimulation of motor cortex in susceptible humans. Brain, 1991; 114: 2505-2520.

61. Cohen LG, Topka H, Cole RA, Hallett M. 1991. Leg paresthesias induced by magnetic brain stimulation in patients with thoracic spinal cord injury. Neurology, 1991; 41:1283-1288.

62. Meyer BU, Diehl R, Steinmetz H, Britton TC, Benecke R. Magnetic stimuli applied over motor and visual cortex: influence of coil position and field polarity on motor responses, phosphenes, and eye movements. Electroencephalography and Clinical Neurophysiology, 1991; 43:121-134.

63. Kammer T. Phosphenes and transient scotomas induced by magnetic stimulation of the occipital lobe: their topographic relationship. Neuropsychologia, 1999; 37:191-198.

64. Kammer T, Beck S, Thielscher A, Laubis-Herrmann U, Topka H. Motor thresholds in humans: a transcranial magnetic stimulation study comparing different pulse waveforms, current directions and stimulator types. Clinical Neurophysiology, 2001; 112:250-258.

65. Cowey A, Walsh V. Magnetically induced phosphenes in sighted, blind and blindsighted observers. Neuroreport, 2001; 11:3269-3273.

66. Amassian VE, Cracco RQ, Maccabee PJ. A sense of movement elicited in paralyzed distal arm by focal magnetic coil stimulation of human motor cortex. Brain Research, 1989; 479:355-360.

67. Brasil-Neto JP, Valls-Sole J, Pascual-Leone A, Cammarota A, Amassian VE, Cracco R, Maccabee P, Cracco J, Hallett M, Cohen LG. Rapid modulation of human cortical motor outputs following ischaemic nerve block. Brain, 1993; 116:511-525.

68. Ellaway PH, Prochazka A, Chan M, Gauthier MJ. The sense of movement elicited by transcranial magnetic stimulation in humans is due to sensory feedback. Journal of Physiology, 2004; 556:651660 .

69. Gurovskiy NN, Bryanov II, Yegorov AD. Changes in the vestibular function during space flight. Acta Astronautica, 1975; 2:207-216.

70. Von Baumgarten RJ, Baldrighi G, Shillinger GL, Harth O, Thuemler R. Vestibular function in the space environment. Acta Astronautica, 1975; 2:49-58.

71. Kornilova LN. Orientation illusions in spaceflight. Journal of Vestibular Research, 1997; 7:429-439.

72. Paqueron X, Leguen M, Rosenthal D, Coriat P, Willer JC, Danziger N. The phenomenology of body image distortions induced by regional anaesthesia. Brain, 2003; 126:702-712.

AU: editors

73. Kircher T, David A. The self in neuroscience and psychiatry. Cambridge University Press, Cambridge. names? 2003.

74. Blanke O. Illusions visuelles. In Neurophtalmologie. Masson, Paris. 2004:147-150. 